\title{
Statistical Analysis of Secondary Electron Spectroscopic Images Using Ultra-Low Energies Reveal Nanoscale Surface Morphology of Functional Organic Blends
}

\author{
Jochen Kammerer ${ }^{1}$, Riva Alkarsifi ${ }^{2}$, Christine Videlot-Ackermann ${ }^{2}$, Olivier Margeat ${ }^{2}$, Bernd Schindler ${ }^{3}$, \\ Irene Wacker ${ }^{1,4}$, Jörg Ackermann ${ }^{2}$, Rasmus R Schröder ${ }^{1,4}$, Martin Pfannmöller ${ }^{1}$ \\ 1. Centre for Advanced Materials, Heidelberg University, Heidelberg, Germany \\ 2. UMR CNRS 7325, CINaM, Aix Marseille Université, Marseille, France \\ 3. Carl Zeiss Microscopy GmbH, Oberkochen, Germany \\ 4. Cryo Electron Microscopy, Heidelberg University / Medical Faculty, Heidelberg, Germany
}

The optimization of blends of functional organic blends, e.g. polymers and fullerenes for organic photovoltaics, has been catalyzed by insights gained through spectroscopic imaging of low energy-loss electrons (EEL-SI) in the transmission electron microscope (TEM) [1]. In previous work, applying nonlinear statistical data analysis to variations in optical excitations three main phases were identified in photoactive blends [2]. The effect of such an analysis of spectroscopic imaging data is based on the reduction of data dimensionality to allow classification of spectra according to physically meaningful signal variations.

In the TEM the collected signal originates from the transmitted beam and provides information along a projected path through the sample. However, local information at interfaces or in z-direction is difficult to obtain. With an SEM, data acquisition can be limited to the surface, particularly when imaging at ultra-low landing energies. In this work we demonstrate that material phases can be visualized at similar resolution to TEM using spectroscopic imaging of secondary electrons (SE-SI) with a novel aberration corrected SEM (Delta-SEM, Carl Zeiss Microscopy) [3]. It is equipped with a Delta Detector ${ }^{\circledR}$ to record spatially resolved SE spectra by successively changing an upstream grid voltage. Correcting imaging aberrations allows to maintain high spatial resolution while using ultra-low primary energy of $50 \mathrm{eV}$.

Fig. 1 shows results from a TEM EEL-SI analysis of a blend of the polymer PTB7 and fullerene PCBM using $60 \mathrm{keV}$ primary energy. Plasmon signals are clearly shifted as seen in the EEL spectra of three phases (Fig. 1a). Locally linear embedding as non-linear statistical analysis generates a visual map of three phases (Fig. 1b). The discrimination is based on contrast differences such as the bright PCBM clusters in Fig. 1c. However, contrast variations remain weak due to the domains' overlapping in 3D. When investigating the same sample by SEM SE-SI using $50 \mathrm{eV}$ primary energy only the surface and a very small area below is probed. Original and intensity-normalized SE spectra of material phases derived from classification are shown in Figure 2a,b. Based on the raw spectra a classification map is generated by using signal discrimination from non-linear statistical analysis (Fig. 2c). In comparison to Fig. 1c, image contrast from the signal near $0 \mathrm{~V}$ counter voltage (Fig. 2d) is more distinct. Furthermore, after normalizing contrast reversal for the material phases is revealed when comparing e.g. the images corresponding to $-16 \mathrm{~V}$ and near $0 \mathrm{~V}$ counter voltage (Fig. 2e,f).

We show that aberration corrected SE-SI in the SEM at ultra-low energies permits to distinguish soft material phases through novel contrast mechanisms. As DELTA-SEM imaging is limited to the surface only, 3D projection artefacts to the phase segmentation is eliminated. Since the damaging cross section decreases at ultra-low landing energies [4], we expect that this method will allow visualizing of a variety of otherwise beam-sensitive materials that are employed for flexible and complex additive systems. 
References:

[1] M Pfannmöller, W Kowalsky and RR Schröder, Energy \& Environmental Science 6 (2013), p. 2871.

[2] M Pfannmöller et al, Nano Letters 11 (2011), p. 3099.

[3] cf. RR Schröder et al, Microscopy and Microanalysis Conference Proceedings of this conference.

[4] P Li and RF Egerton, Microscopy and Microanalysis 9 (2003), p. 986.

[5] The authors acknowledge funding of the DELTA project by the ministry of Research and Education to R.R.S (FKZ: 13GW0044), and funding of J.K. and M.P. by the ministry of research, Baden-

Württemberg, through the HEiKA materials research centre FunTECH-3D.
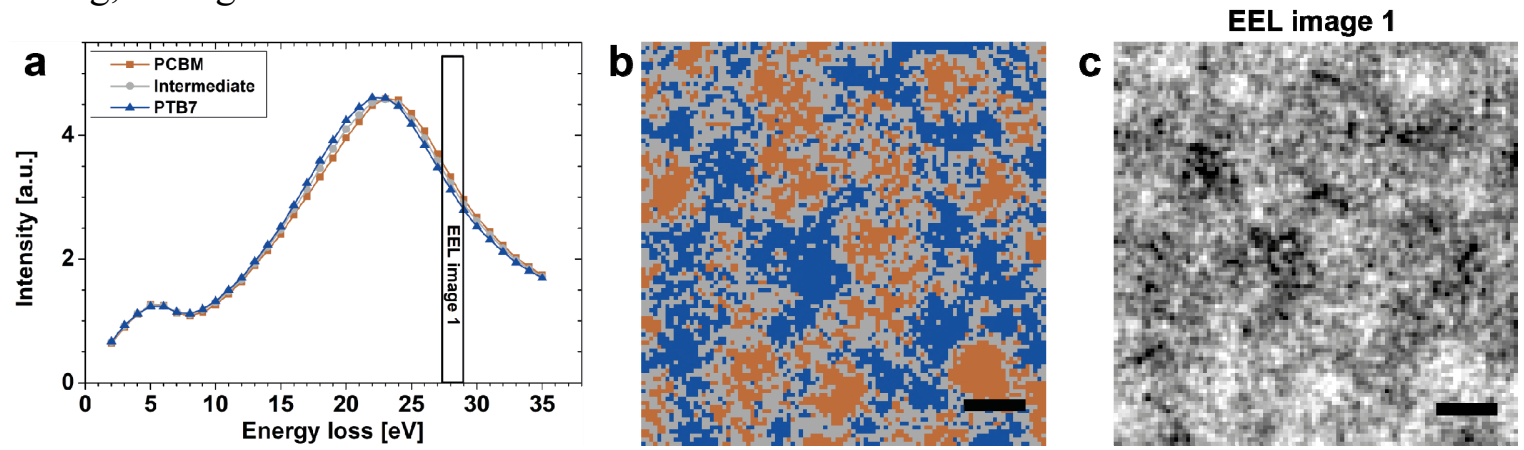

Figure 1. Discrimination of fullerene (PCBM), polymer (PTB7) and intermediate phases using TEM EEL-SI data sets based on differences in plasmon excitations (a). Non-linear statistical analysis uses these discriminating signals for segmentation resulting in a map of nanoscale phases (b). An example image recorded around $27 \mathrm{eV}$ illustrates material contrast obtained from the bulk of the sample (c) by projecting the entire 3D information into one 2D image. Scale bars $50 \mathrm{~nm}$.
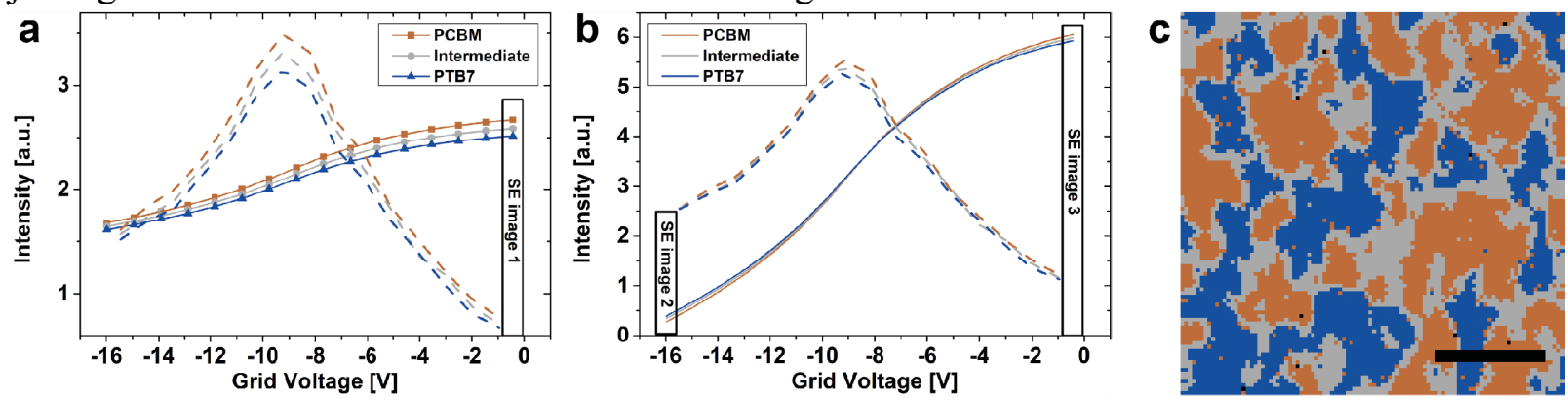

SE image 1
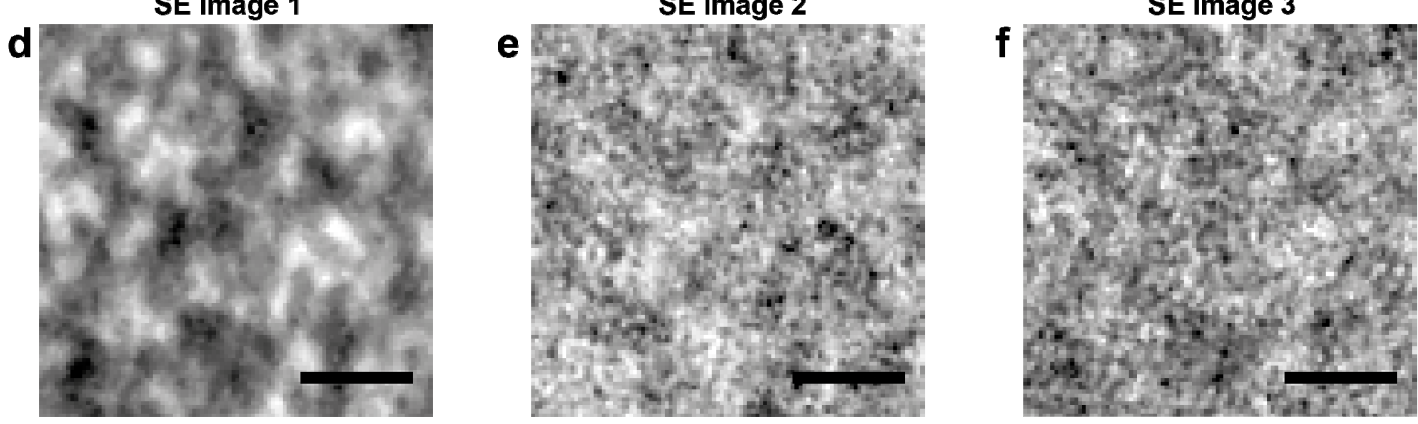

Figure 2. Raw (a) and normalized (b) SE-SI data sets recorded at $50 \mathrm{eV}$. Accumulated SE spectra (solid lines) and their derivatives (dashed lines) have different intensities for the three phases, which were identified by non-linear statistical analysis. The map of the three phases (c) shows a similar morphology as in Figure 1 but from the sample surface. Underlying image contrast from absolute (d) and normalized $(\mathrm{e}, \mathrm{f})$ intensities demonstrate the typical contrast inversion as in TEM EEL-SI. Scale bars $50 \mathrm{~nm}$. 